\title{
MECHANICAL PROPERTIES OF PARTS OF MEDICAL COMPONENTS PRODUCED USING ADDITIVE MANUFACTURING TECHNOLOGIES
}

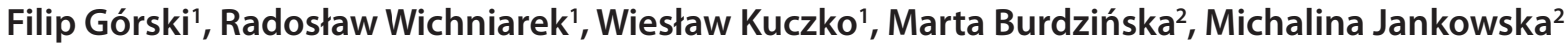 \\ 1 Poznan University of Technology, Chair of Production Engineering and Management, Poznan, Poland, e-mail: \\ filip.gorski@put.poznan.pl,wieslaw.kuczko@put.poznan.pl, radoslaw.wichniarek@put.poznan.pl \\ 2 ArjoHuntleigh Polska Sp. z o. o., ul. Ks Wawrzyniaka 2, 62-052 Komorniki, e-mail: marta.burdzinska@gmail. \\ com, jankowska_michalina@wp.pl
}

Received: 2017.04.14

Accepted: 2017.05.05

Published: 2017.06.01

\begin{abstract}
The paper presents results of tests conducted on the elements of medical devices slings used in medical lifts - manufactured using additive technologies. The project assumed to produce 100 samples of clips with varying design, material and orientation parameter. Samples were manufactured using FDM and SLA processes and then tested for mechanical strength, load transmission and functionality, using certified equipment. The paper shows full methodology and the obtained test results.
\end{abstract}

Keywords: additive manufacturing, slings, mechanical properties, medical devices.

\section{INTRODUCTION}

Additive manufacturing (widely known as 3D printing) and rapid prototyping have found many applications in medicine $[10,7]$. They are used wherever there is a need of an individualized solution, as they allow fast production of a demanded shape with no tooling [2]. Examples of medical use include treatment of injuries of ankles or wrists [6] or orthodontic tooling [9]. There is also a group of personalized medical products that are not used directly by a patient, but instead by the surgeon before or during the operation, for example templates for bone cutting [11]. Attempts have also been made to use additive manufacturing technologies for production of medical instruments used during operations [8].

Two most widespread additive manufacturing technologies are Fused Deposition Modelling (FDM) and stereolithography (SLA). The manufacturing in the FDM technology is carried out by extrusion of heated thermoplastic material (usually ABS or PLA) through the nozzle in the head, which moves in the XY plane. The material is deposed layer by layer - after one layer is finished, the table moves in $\mathrm{Z}$ direction and the process of material extrusion continues until the shape is finished. The SLA process is based on curing photopolymers with UV light delivered by a low-power laser. The laser moves in XY plane and hardens a liquid polymer, after one layer is finished, the table moves vertically, just as in the FDM technology. In both processes, information about geometry of consecutive layers is sent to a numerically controlled machine directly from the digital representation of the product, stored in a CAD model [2]. Both processes can be realized on low-cost machines (priced below $\$ 1,500$ ).

A final product manufactured using any additive manufacturing technology can be characterized by some coefficients, which are influenced by many factors [1]. Unlike in most manufacturing technologies, values of parameters of the additive manufacturing process can be more significant than the properties of the part material two different sets of process parameters applied to the same geometry can result in obtaining two products of entirely different properties [4]. Each set of process parameters: orientation of the product in the working chamber, layer thickness and 
method of filling of the layer contour, will make the part structure look different, which will result in different values of coefficients such as strength, accuracy or surface quality $[5,3]$.

All the above mentioned limitations are particularly important when a durable, accurate and repeatable product is to be obtained. In medical branch, an example of such a product can be slings of medical lifts, which belong the most frequently used medical devices worldwide, helping persons who have problems with performing daily activities [12]. The paper presents studies answering if clips of these slings can be manufactured additively by popular technologies and if the additive manufacturing can be used for verification of the prototypes of new design of such devices. All the studies were performed in a laboratory of a company manufacturing medical lifts.

\section{MATERIALS AND METHODS}

\section{Main concepts of research}

The main aim of the paper was to verify if it is possible to use additive manufacturing technologies (widely known as 3D printing) for production of clips of slings for medical lifts. To achieve this aim, it was decided to manufacture samples of this part using two different processes - stereolithography and Fused Deposition Modelling, three different materials - photohardening resin in SLA, ABS and PLA in FDM - and different process parameters (orientations in working chamber during layer deposition). The clips were prepared using an original design used in existing lifts.

The second aim of the studies presented in the paper was to check if the use of additive manufacturing technologies is an effective way of assessment of a new project of a sling. Modification of an original model of a part was proposed (Fig. 1) to increase safety of use of a sling by more stable mounting on a lift hanger. To verify mechanical

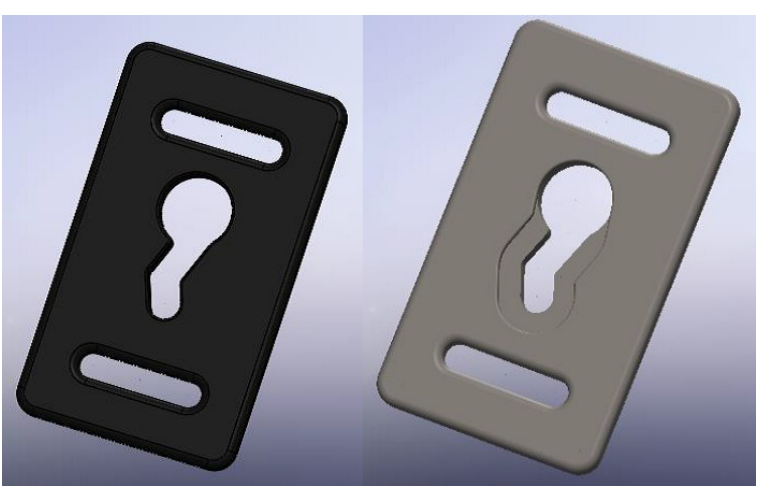

Fig. 1. Original (left) and modified (right) design of a clip for slings in medical lifts

and utility properties of manufactured clips, they were subjected to tests conforming with study protocols of companies manufacturing these elements [12] and were compared to the properties of the original elements. All the studies were performed in a certified industrial laboratory.

\section{Additive manufacturing processes}

According to the main concept presented above, on the basis of designed 3D models, a total amount of 100 samples was manufactured using low-cost additive manufacturing technologies. A variety of clips was determined by the following properties: process, material, design and orientation of a sample in working chamber of a given additive manufacturing machine during the layer deposition process. Variants of the samples and process parameters, depending on the used process, are presented in Table 1. For each of 5 configurations, 10 samples were manufactured, both for the original and the new design.

In the FDM process, support structures were used. For the ABS material, it was the HIPS (High Impact Polystyrene) extruded by the second nozzle of the machine, while for the PLA material only one nozzle was used. The support was made out of the same material as the samples. It was due to processing conditions

Table 1. Variants of manufactured samples regarding process parameters

\begin{tabular}{|c|c|c|c|c|c|}
\hline Used process & \multicolumn{4}{|c|}{ Fused Deposition Modelling } & Stereolithography \\
\hline Machine & \multicolumn{4}{|c|}{ Stratasys MakerBot Replicator 2X } & XYZPrint Nobel 1.0 \\
\hline Build material & \multicolumn{2}{|c|}{ ABS } & \multicolumn{2}{|c|}{ PLA } & Photohardening resin \\
\hline Layer thickness & \multicolumn{2}{|c|}{$0,3 \mathrm{~mm}$} & \multicolumn{2}{|c|}{$0,3 \mathrm{~mm}$} & $0,1 \mathrm{~mm}$ \\
\hline Temperatures: build/support/table & \multicolumn{2}{|c|}{$230 / 250 / 110^{\circ} \mathrm{C}$} & \multicolumn{2}{|c|}{$215 / 215 / 60^{\circ} \mathrm{C}$} & n/a (ambient) \\
\hline Build orientation & Flat & Side & Flat & Side & Flat \\
\hline Average build time & $52 \mathrm{~min}$. & $165 \mathrm{~min}$. & $40 \min$. & $90 \mathrm{~min}$. & $80 \mathrm{~min}$. \\
\hline
\end{tabular}




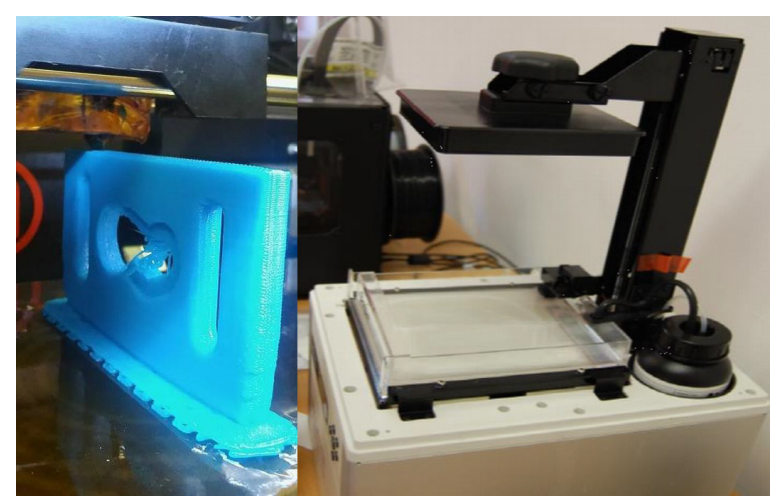

Fig. 2. Manufacturing clips using low-cost FDM (left) and SLA (right)

- much different temperatures of processing of HIPS and PLA prevent using them together in one 3D print. For both technologies, only materials recommended and supplied by machine producers were used in manufacturing and postprocessing. Realization of processes is shown in exemplary photographs in Fig. 2.

In case of the FDM process, the only form of post-processing (after removal of samples out of the machine) was removal of support and raft. In case of the SLA process, post-curing was necessary - it was realized by exposing samples to concentrated UV light over the period of approx. 20-30 minutes, right after removal out of the machine and cleaning.

\section{Testing procedure}

The tests performed on the manufactured parts were divided into strength tests, loading tests and use tests. The strength tests were tension and bending, performed according to standards by the ArjoHuntleigh company [13]. The tests were performed using the Inspect 10 Table machine by Hegewald \& Peschke company. The loading tests were performed using a dedicated, special testing machine, designed according to requirements contained in the EN-PN 10535:2002 standard. The use tests were performed on a mobile medical lift Maxi Twin by the ArjoHuntleigh company.

Environmental conditions of all the tests were as following:

- ambient temperature $-20 \pm 5^{\circ} \mathrm{C}$,

- air humidity - 30-65\%,

- atmospherical pressure - 950 - 1050 mbar.

The bending test consists in placing a clip in special jaws of the testing machine and then gradual bending using a minimum force value of $5884 \mathrm{~N}$, until bending of $90^{\circ}$ is achieved. The clip is verified

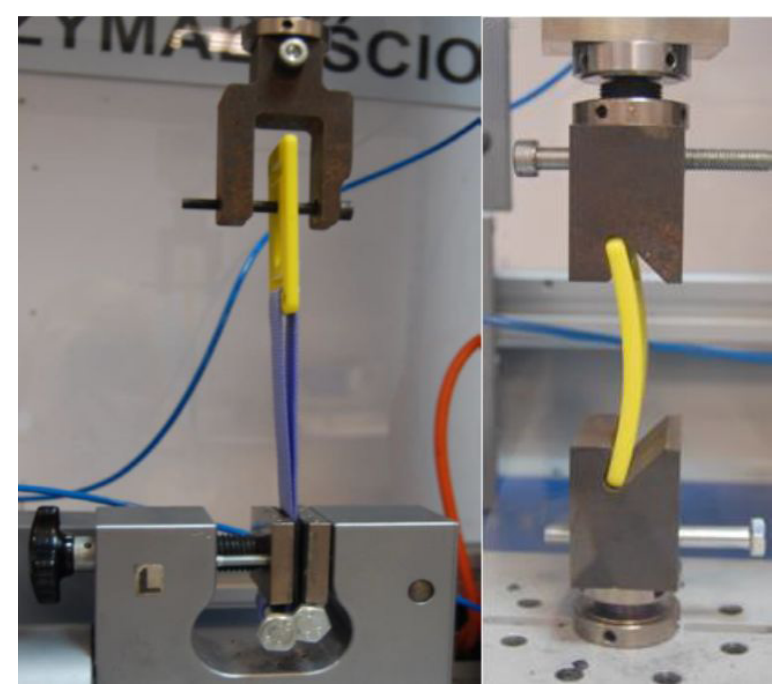

Fig. 3. Tension and bending tests of manufactured clips

positively if there are no visible fractures after keeping it in maximal flexure at least for 3 seconds [13].

In the tension test, a clip is mounted to jaws of a testing machine in such a way, that its upper part is hanging on a pin going through the central hole of a clip, while the lower jaws hold a belt going through the slit in the base of a clip. This test is a static tension test - a mounted clip is gradually tensed, with velocity of $25 \mathrm{~mm} / \mathrm{min}$. A clip should resist tension of the force of at least 4413 $\mathrm{N}$. If it does not fail at this value of force - the test is passed [13]. Figure 3 presents strength tests of exemplary samples.

The loading test of a sling is conforming with the EN-PN 10535:2002 standard requirements and allows determining if a given sling fulfills criteria of acceptability. In the presented work, the studies were performed on the Flites slings by ArjoHuntleigh (Fig. 4). The test consists in loading the fully prepared set of a sling with four clips up to the value of 1,5 SWL (safe working load). The Flites slings are loaded over the time of 20 minutes, with SWL equal to $408 \mathrm{~kg}$.

The use tests consisted in simulation of standard activities happening during care of a patient using a lift. These tests consisted in, among other things, placing a mannequin in a sling and then lifting, lowering and transporting it. Moreover, tests with volunteers were performed (Fig. 4) they were placed in a lift and then simulated specific actions by a patient (e.g. convulsions, panic attack, epilepsy attack, fainting etc.).

After the loading and use tests, visual inspection was a method of checking if during the test any of four clips or a whole sling was damaged. 


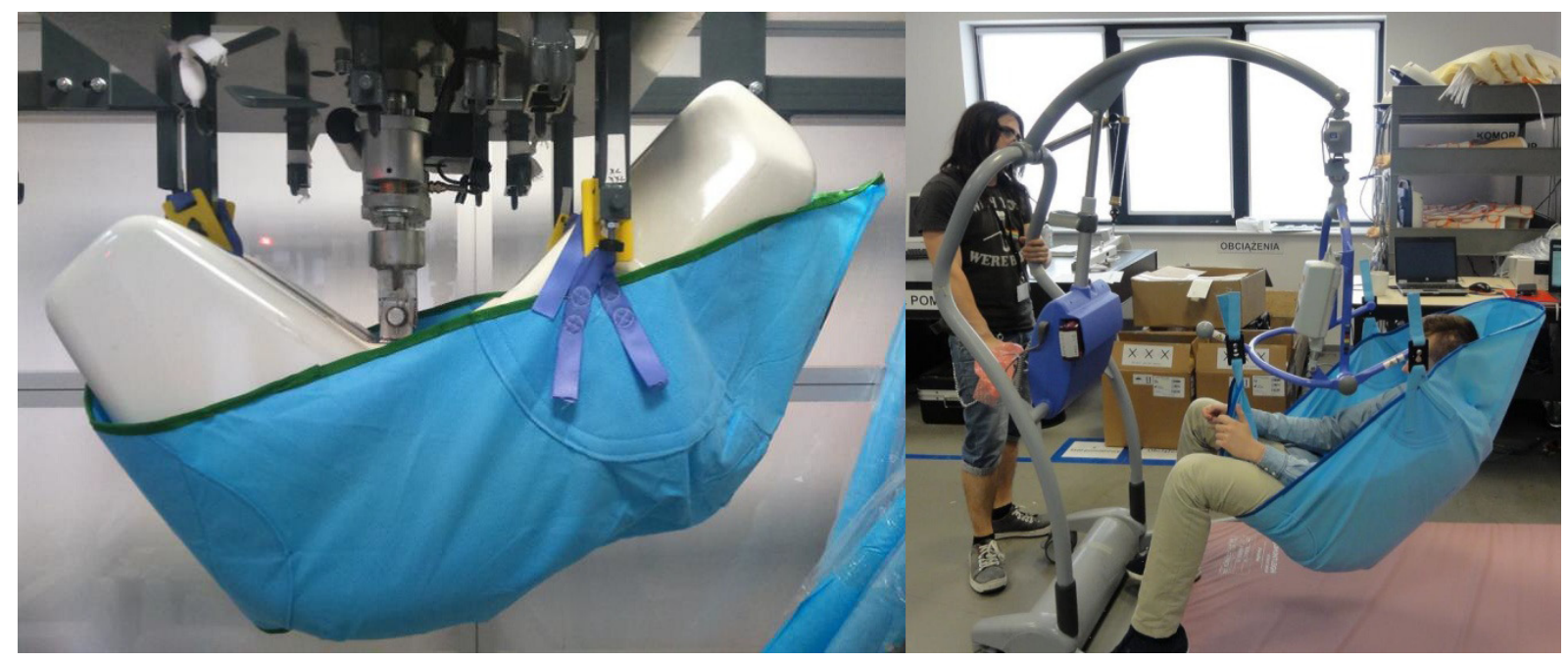

Fig. 4. Loading and usability tests

In case of visible damage of material, stitch or a clip itself, a given test is marked as failed [13].

\section{RESULTS AND DISCUSSION}

The results of all tests are presented in Table 2. Reference, injection molded clip results are also presented. Figure 5 presents examples of damaged samples after both tests.

The following observations were made during the tests and after the analysis of results:

- no sample manufactured additively passed all the tests - the best samples survived loading and use tests, but failed on tension and bending tests, due to insufficient plasticity and tension strength,
- the tests allowed to find that the new design of a clip, proposed by the authors, helped increasing both mechanical properties and usability - assembly of elements in the used lift was more stable and better fitting was obtained,

- clips - parts of rectangular shape with relatively low thickness - if manufactured additively, should be made of ABS and in flat orientation, PLA material is too rigid (it fractures easily, it is harder to bend - requires higher force), while side orientation for this type of part does not allow obtaining large enough accuracy and strength,

- samples made out of the photohardening resin in the low-cost SLA process were the worst in terms of strength, there were also certain prob-

Table 2. Tests results

\begin{tabular}{|c|c|c|c|c|c|c|c|}
\hline No. & $\begin{array}{l}\text { Process, material, } \\
\text { orientation }\end{array}$ & Design & $\begin{array}{l}\text { Avg. max tension } \\
\text { force }[N]\end{array}$ & $\begin{array}{l}\text { Avg. max bending } \\
\text { force }[\mathrm{N}]\end{array}$ & $\begin{array}{l}\text { Strength } \\
\text { test }\end{array}$ & $\begin{array}{l}\text { Loading } \\
\text { test }\end{array}$ & Use test \\
\hline 1. & FDM/ABS/flat & \multirow{5}{*}{$\begin{array}{l}\text { old } \\
\text { (orig.) }\end{array}$} & 1490 & 959 & \multirow{10}{*}{ fail $^{*}$} & pass & pass \\
\hline 2. & FDM/ABS/side & & 1032 & 1183 & & fail ${ }^{*}$ & pass \\
\hline 3. & FDM/PLA/flat & & 2623 & 2653 & & pass & pass \\
\hline 4. & FDM/PLA/side & & 1621 & 2476 & & fail $^{*}$ & fail $^{* *}$ \\
\hline 5. & SLA/resin/flat & & 608 & 997 & & fail $^{*}$ & fail $^{* *}$ \\
\hline 6. & FDM/ABS/flat & \multirow{5}{*}{ new } & 2420 & 2278 & & pass & pass \\
\hline 7. & FDM/ABS/side & & 925 & 1825 & & fail $^{*}$ & pass \\
\hline 8. & FDM/PLA/flat & & 3354 & 3573 & & pass & pass \\
\hline 9. & FDM/PLA/side & & 2923 & 3502 & & fail $^{*}$ & fail $^{* *}$ \\
\hline 10. & SLA/resin/flat & & 731 & 1904 & & fail $^{*}$ & fail $^{* *}$ \\
\hline 11. & Injection mold & old & 4564 & 840 & pass & pass & \\
\hline
\end{tabular}

* mechanical failure before acceptable load / time reached

** assembly problems - insufficient accuracy, small mechanical damages 

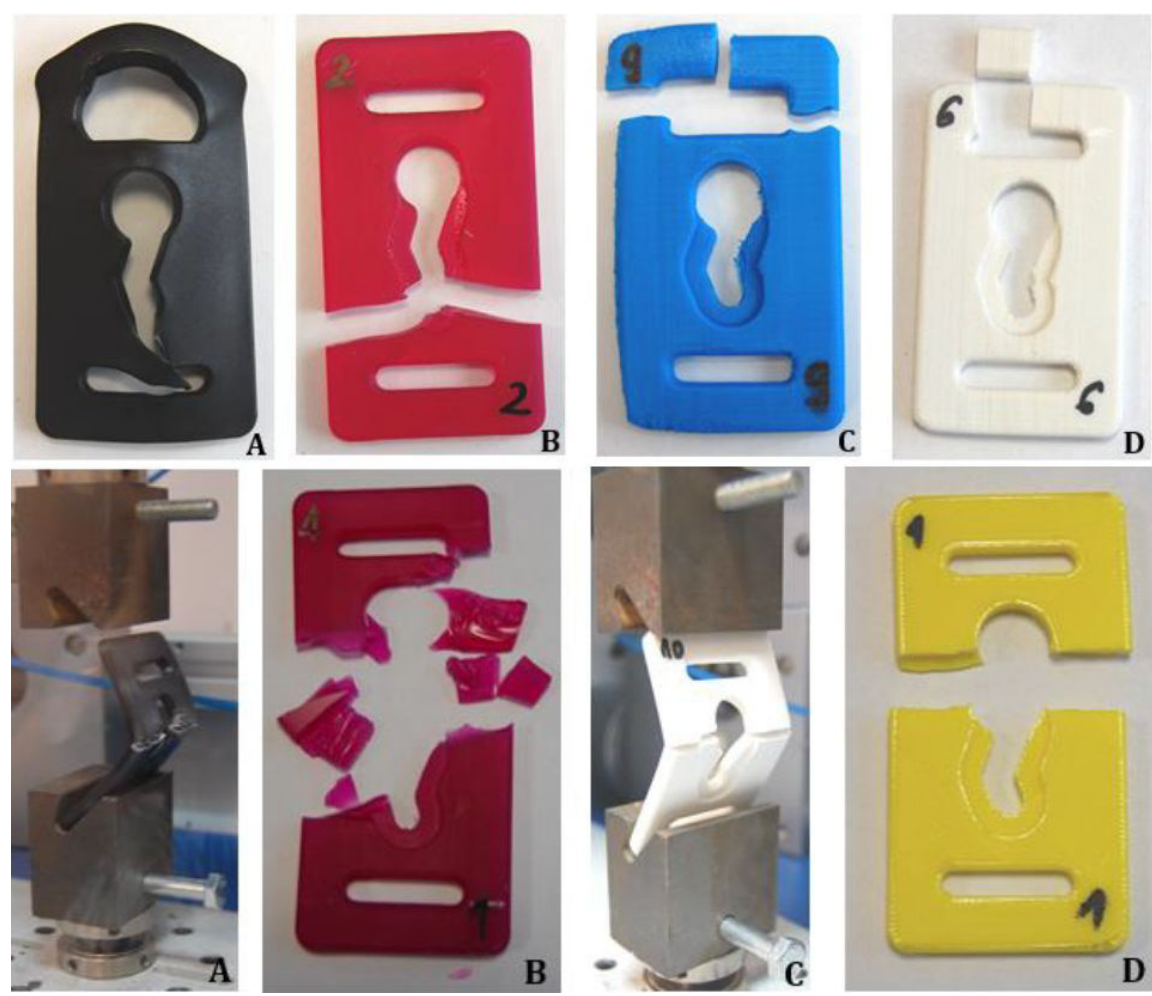

Fig. 5. Clips destroyed in tension tests (top) and bending tests (bottom)

lems with accuracy (revealed in the assembly process at the stage of usability tests), despite layer thickness being much lower than in the FDM process,

- taking all the processing parameters (process, material, orientation) into account, the general rule applies that the best results were achieved by the cheapest variant, which is the FDM process, flat orientation and the ABS material (total production cost of approx. 70 PLN per part for both designs), while the worst - by the most expensive variants (ABS, side orientation - approx. 215 PLN and SLA - approx. 240 PLN per part for both designs),

- the original sample, made out of polypropylene, achieved far better results in all tests; however, the use of polypropylene in the FDM process is difficult, but this should be investigated in further studies.

\section{CONCLUSIONS}

The work presented in the paper allowed to confirm the general, common knowledge about the cheap 3D printing processes: they are suitable for prototyping purposes, but in terms of maintaining high level of mechanical properties and accuracy, they do not fit into the medical industry standards. The authors believe that such tests should still be performed using various processes and materials to find more practical applications of 3D printed parts in medicine. A general conclusion, as regards mechanical properties of the additively manufactured polymer parts, is that they do not present as good plastic behavior as their injection molded counterparts do - all the manufactured clips behaved as if made of rigid materials, presenting very small plastic deformation under load. This also applies to impact strength. While planning the use of $3 \mathrm{D}$ printed polymer parts in functional devices, one should have that observation in mind - the FDM or SLA processes do not allow obtaining parts with mechanical characteristics equal to those of the raw material.

The two investigated cheap additive manufacturing technologies - FDM and SLA - both have advantages and disadvantages. The FDM process realized on low-cost machines such as the MakerBot Replicator 2X requires supervision, generates many errors and, in many cases, does not allow obtaining functional, complete part in a first approach. During the tests, there were certain problems with process stability, especially for the PLA material. The SLA process, 
on the other hand, does not require supervision at all. It produces parts with relatively smooth surface and, in theory, allows obtaining better accuracy. However, it takes long time, post-processing is labor consuming and the obtained accuracy is not always better than that of the FDM process, as proved in the tests performed for the purpose of this paper.

To sum up, the concept of manufacturing durable parts for medical lifts using additive technologies was disproven. However, usability of these technologies for prototyping and manufacturing of short series of experimental parts was proven to be worth the effort. The future studies should focus on testing different materials and process parameters to obtain the best mechanical properties and accuracy coefficients.

\section{REFERENCES}

1. Bellini A. and Guceri S. Mechanical characterization of parts fabricated using Fused Deposition Modeling. Rapid Prototyping Journal, 9, 2003, 252 - 264.

2. Chua C. K., et al. Rapid Prototyping: Principles and Applications. World Scientific Publishing, 2010.

3. Gajdoš I., et al. Structure and tensile properties evaluation of samples produced by Fused Deposition Modeling. Open Engineering, 1(6), 2016, 86-89.

4. Górski F., et al. Computation of Mechanical Properties of Parts Manufactured by Fused Deposition Modeling using Finite Element Method. 10th International Conference on Soft Computing Models in Industrial and Environmental Applications,
Advances in Intelligent Systems and Computing, 368, 2015, 403-413.

5. Górski F., et al. Strength of ABS parts produced by fused deposition Modeling technology - a critical orientation problem. Advances in Science and Technology Research Journal, 9, 2015, 12-19.

6. Kim H., Jeong S. Case study: Hybrid model for the customized wrist orthosis using 3D printing. Journal of Mechanical Science and Technology, 29(12), 2015, 5151-5156.

7. Marro A., et al. Three-Dimensional Printing and Medical Imaging: A Review of the Methods and Applications. Current Problems in Diagnostic Radiology, 45, 2016, 2-9.

8. Rankin T.M., et al. Three-dimensional printing surgical instruments: are we there yet?. Journal of Surgical Research, 189(2), 2014, 193-197.

9. Salmi M., et al. Rapid Tooling Method for Soft Customized Removable Oral Appliances. The Open Dentistry Journal, 6, 2012, 85-89.

10. Syam W. P., et al. Rapid prototyping and rapid manufacturing in medicine and dentistry. Virtual and Physical Prototyping, 6(2), 2011, 79-109.

11. Tukuru N., et al. Rapid prototype technique in medical field. International Journal of PharmTech Research, 1(4), 2008, 341-344.

12. Website: ArjoHuntleigh Product, Patient Transfer Solutions Brochure; http://www.arjohuntleigh. com/ (access: 15.03.2017).

13. Website: ArjoHuntleigh Test Methods for Verification or Validation. Verification of Clip for batch release, HMX492. Document number 100007316; http:// www.arjohuntleigh.com/ (access: 15.03.2017). 